\title{
CHARACTERISTIC NUMBERS OF UNITARY TORUS-MANIFOLDS
}

\author{
BY PETER LÖFFLER \\ Communicated by Glen Bredon, April 3, 1973
}

1. Introduction. Unitary torus-manifolds have been studied by Hamrick and Ossa in [5]. They show that the bordism class of such a manifold is determined by its fixed point set. In [4] tom Dieck introduced homotopical bordism theories. Now the question arises: Is a result corresponding to the one of Hamrick and Ossa true for homotopical bordism? The answer is given in Proposition 2.3. From this proposition we get the following

THEOREM. Unitary torus-manifolds are determined by K-theory characteristic numbers

The details of all proofs are contained in [6], the author's thesis, which was written under T. tom Dieck.

2. Characteristic numbers. There are two ways of defining what an equivariant unitary $G$-manifold should be. The first is given by stabilizing the tangent bundle with $\boldsymbol{R}^{n}$ (trivial $G$-action), the second by stabilizing with complex representations. We denote the bordism theories so obtained by $\overline{\mathfrak{U}}_{*}^{G}$ and $\mathfrak{U}_{*}^{G}$, respectively. There is an obvious natural transformation $j: \overline{\mathfrak{U}}_{*}^{G} \rightarrow \mathfrak{U}_{*}^{G}$ of equivariant homology theories.

LEMMA 2.1. $j$ is a monomorphism for compact abelian Lie groups.

In [4] a homotopical bordism theory is defined, using equivariant Thom spectra. There exists a Pontrjagin-Thom construction $i: \mathfrak{U}_{*}^{G} \rightarrow U_{*}^{G}$.

Proposition 2.2. If $G$ is a compact abelian Lie group, then $i$ is injective.

Let $S$ denote the multiplicatively closed set in $U_{*}^{G}$ generated by the Euler classes of finite dimensional complex representations. Let $\lambda: U_{*}^{G} \rightarrow S^{-1} U_{*}^{G}$ denote the localization map. As forming $S^{-1} U_{*}^{G}$ corresponds to "restriction to the fixed point set" we have in analogy to [5].

Proposition 2.3. $\lambda$ is injective iff $G$ is a torus.

Let $E G$ denote a free contractible $G$-space, $B G=E G / G$, the projection

AMS(MOS) subject classifications (1970). Primary 57D85.

Key words and phrases. Equivariant bordism. 
$E G \rightarrow$ point induces in unitary cobordism

$$
\alpha: U_{G}^{*} \rightarrow U_{G}^{*}(E G) \cong U^{*}(B G) ;
$$

$\alpha$ is in fact a natural transformation of equivariant cohomology theories [4].

Proposition 2.4. $\alpha$ is injective for a torus.

Proof. By using Proposition 2.3 one only has to show that $S^{-1} \alpha$ is injective. This can be reduced to showing this for a group of the form $Z_{p}$, $p$ a prime. This was proved in [3].

Let $p: U_{G}^{*} \rightarrow U^{*}$ be "forgetting the $G$-action". We denote the kernel by $I_{G}$. In analogy to $[1]$ we have

Proposition 2.5. Let $G$ be a compact abelian Lie group or a group admitting a free complex representation. Then $\alpha$ induces an isomorphism

$$
\hat{\alpha}:\left(U_{G}^{*}\right) \stackrel{\wedge}{\rightarrow} U^{*}(B G)
$$

where $\widehat{\text { denotes }} I_{G}$-adic completion, provided $U_{G}^{\text {odd }}=0$.

Let us denote by $B: U^{*}(X) \rightarrow K^{*}(X)\left[a_{1}, a_{2}, \ldots\right]$ the Boardman map [2]. This is known to be split injective for $X$ a point. This gives

LEMma 2.6. The map $B: U^{*}(B G) \rightarrow K^{*}(B G)\left[\left[a_{1}, a_{2}, \ldots\right]\right]$ is injective for $G$ a torus.

If we look at the composition $B \circ \alpha \circ i$ then 2.2, 2.4 and 2.6 lead to

THEOREM 2.7. K-theory characteristic numbers determine the bordism class of a unitary torus manifold.

\section{REFERENCES}

1. M. F. Atiyah, Characters and cohomology of finite groups, Inst. Hautes Êtudes Sci. Publ. Math. No. 9 (1961), 23-64. MR 26 \#6228.

2. Th. Bröcker and T. tom Dieck, Kohordismentheorie, Lecture Notes in Math., vol. 178, Springer-Verlag, Berlin and New York, 1970. MR 43 \# 1202.

3. T. tom Dieck, Periodische Abbildungen unitärer Mannigfaltigkeiten, Math. Z. 126 (1972), 275-295.

4. - Bordism of G-manifolds and integrality theorems, Topology 9 (1970), 345-358. MR 42 \#1148.

5. G. Hamrick and E. Ossa, Unitary bordism of monogenic groups and isometries, Proc. Second Conf. on Compact Transformation Groups (Amherst, 1971), Lecture Notes in Math., vol. 298, Springer-Verlag, Berlin and New York, 1972, pp. 172-182.

6. P. Löffler, Thesis, Saarbrücken, 1973.

Mathematisches Institut, Universitat des Saarlandes, 66 SaArbrucken, Federal RePUBLIC OF GERMANY 